\title{
Enhanced Electrocatalytic Oxygen Evolution in Au-Fe Nanoalloys
}

\author{
Irene Vassalini, Laura Borgese, Michele Mariz, Stefano Polizzi, Giuliana Aquilanti, \\ Paolo Ghigna, Andrea Sartorel, Vincenzo Amendola,* and Ivano Alessandri*
}

\begin{abstract}
Oxygen evolution reaction (OER) is the most critical step in water splitting, still limiting the development of efficient alkaline water electrolyzers. Here we investigate the OER activity of $\mathrm{Au}-\mathrm{Fe}$ nanoalloys obtained by laser-ablation synthesis in solution. This method allows a high amount of iron (up to 11 at \%) to be incorporated into the gold lattice, which is not possible in $\mathrm{Au}-\mathrm{Fe}$ alloys synthesized by other routes, due to thermodynamic constraints. The $A u_{0.89} F_{0.11}$ nanoalloys exhibit strongly enhanced OER in comparison to the individual pure metal nanoparticles, lowering the onset of $O E R$ and increasing up to 20 times the current density in alkaline aqueous solutions. Such a remarkable electrocatalytic activity is associated to nanoalloying, as demonstrated by comparative examples with physical mixtures of gold and iron nanoparticles. These results open attractive scenarios to the use of kinetically stable nanoalloys for catalysis and energy conversion.
\end{abstract}

$T_{\text {he intrinsic slow kinetics of oxygen evolution reaction }}$ (OER) represents a major obstacle towards the development of efficient electrochemical or photoelectrochemical cells for water splitting. ${ }^{[1]}$ In alkaline media, OER occurs through the consumption of hydroxy ions to produce oxygen and water $\left(4 \mathrm{OH}^{-} \rightleftarrows \mathrm{O}_{2}+2 \mathrm{H}_{2} \mathrm{O}+4 \mathrm{e}^{-}\right)$. Various combinations of metals, oxides, mixed-metals and alloys have been investigated as catalysts to improve OER kinetics. ${ }^{[2]}$ In particular, mixedmetal or alloy electrodes are attracting great interest as viable alternatives to the expensive $\mathrm{Ru}$ and Ir-based catalysts. ${ }^{[3-5]}$ In this context, iron is gaining ever-increasing interest as an

[*] Dr. I. Vassalini, Dr. L. Borgese, Prof. I. Alessandri

INSTM and University of Brescia, Mechanical and Industrial

Engineering Department (DIMI)

via Branze 38, 25123 Brescia (Italy)

E-mail: ivano.alessandri@unibs.it

M. Mariz, Prof. A. Sartorel, Prof. V. Amendola

INSTM and Department of Chemical Sciences, University of Padova

via Marzolo 1, 35131 Padova (Italy)

E-mail: vincenzo.amendola@unipd.it

Prof. S. Polizzi

Department of Molecular Sciences and Nanosystems, Centro di Microscopia Elettronica G. Stevanato, Università Ca' Foscari Venezia and INSTM UdR Venezia

30172 Venezia-Mestre (Italy)

Dr. G. Aquilanti

Elettra-Sincrotrone Trieste

s.s. 14, km 163.5, 34149 Basovizza, Trieste (Italy)

Prof. P. Ghigna

INSTM and Department of Chemistry, University of Pavia

viale Taramelli 13, 27100 Pavia (Italy)

(1D)

Supporting information and the ORCID identification number(s) for the author(s) of this article can be found under:

https://doi.org/10.1002/anie.201703387.
OER active component, either alone or in combination with other metals. ${ }^{[6,7]}$ Various research groups demonstrated that $\mathrm{Fe}^{3+}$ plays a key role in OER. ${ }^{[8-15]}$ This fact was not clearly realized before, because the observation of a significant improvement of OER activity was hampered by the poor conductivity of the OER-active material containing the $\mathrm{Fe}^{3+}$ species, typically FeOOH$\cdot{ }^{[14]}$ On the other hand, lower values of overpotential and remarkable catalytic efficiency were observed in the case of $\mathrm{Fe}^{3+}$ species combined with more conductive host materials, like $\mathrm{CoOOH}$ or $\mathrm{NiOOH} .{ }^{[1-15]}$ Two recent papers reported a significant improvement of OER activity in alkaline conditions when the surface of gold was doped with $\mathrm{Fe}^{3+}$ species. ${ }^{[14,15]}$ However, these electrodes are formed upon alkaline oxidation of Au surfaces and subsequent adsorption of $\mathrm{Fe}^{3+}$ OER active sites, which does not allow a precise control of iron-loading and overall chemical composition. The formation of $\mathrm{Au}-\mathrm{Fe}$ nanoalloys could provide an interesting alternative for obtaining high surface area $\mathrm{Au}-\mathrm{Fe}$ OER-active materials with a controlled composition and, in general, new catalysts for different types of reactions, as suggested by Martirez and Carter. ${ }^{[16]}$ The main issue related to $\mathrm{Au}-\mathrm{Fe}$ alloys is the low miscibility of the two metals. Their thermodynamic stability in ambient conditions is indeed restricted to a low Fe content $(<2.5$ at \% $),{ }^{[17]}$ which does not allow for a significant enhancement of the OER activity.

Here we report the first experimental observation of the enhancement of OER in Au-Fe nanoalloys achieved by laserablation synthesis in solution (LASiS), which enables the production of kinetically stable and homogeneous nanoalloys with content of Fe well above the thermodynamic limit (up to 11 at \% ), thanks to the fast kinetics of nanoparticles formation (Figure 1a and Figure S1 in the Supporting Information).

Importantly, LASiS is a "one-step", easily scalable process, which starts from an already formed kinetically stable $\mathrm{Au}-\mathrm{Fe}$ bulk alloy target. ${ }^{[18]}$ The obtained nanoalloys retain the face centred cubic (fcc) structure of $\mathrm{Au}$, though with $\mathrm{Fe}$ atoms as substitutional dopants (Figure $1 \mathrm{~b}-\mathrm{d}$ ). XANES (X-ray absorption near edge structure) spectra collected at the Fe-K and Au- $\mathrm{L}_{\mathrm{III}}$ edges (Figure 1e,f, respectively), and the corresponding derivatives (Figures $1 \mathrm{~g}, \mathrm{~h}$ ), show that iron electronic structure is heavily modified in the nanoalloy, while the gold fingerprint remains very similar to that of the pure metal. At Fe K-edge the onset of absorption is at $7112 \mathrm{eV}$ corresponding to that of metallic iron (Figure $1 \mathrm{~g}$ and inset of Figure 1e). This evidences a significant contribution of metal $\mathrm{Fe}$ states near the Fermi level, as typically observed for the alloys of noble metals with fcc structure with iron. ${ }^{[38]}$

The formation of an intermetallic alloy is further confirmed by the EXAFS (extended X-ray absorption fine 

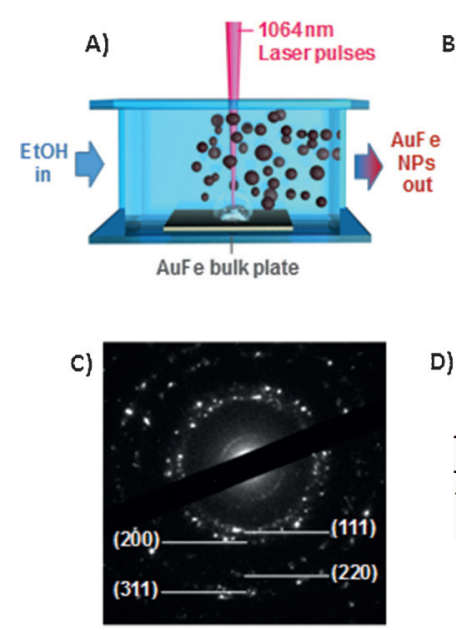
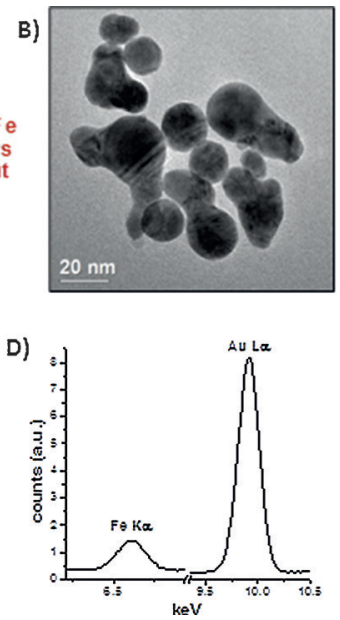

E)

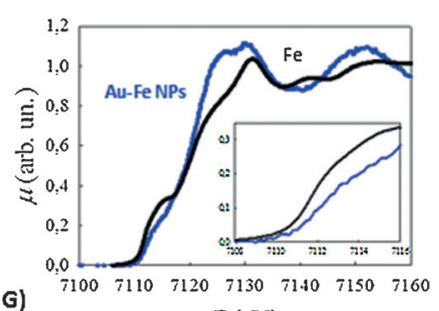

G)

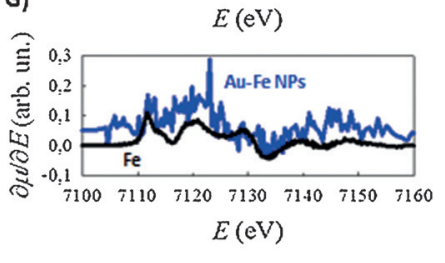

F)

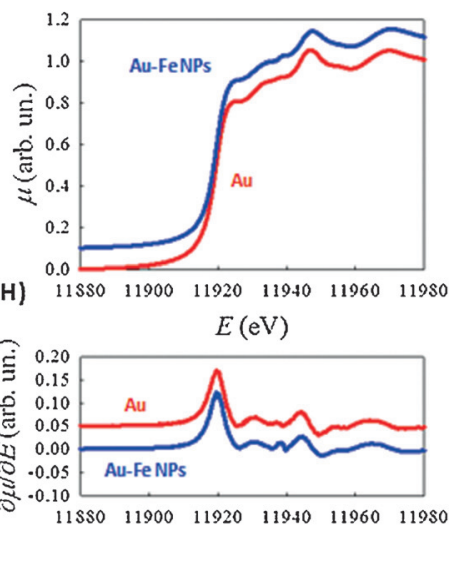

Figure 1. A) Sketch of LASiS of Au-Fe NPs. B-D) Transmission electron microscopy analysis of $\mathrm{Au}_{0.89} \mathrm{Fe}_{0.11}$ nanoalloys: B) representative bright field image of alloy NPs; C) selected area electron diffraction pattern, showing typical reflection of the face-centred cubic cell of these alloys; D) energy dispersive $\mathrm{X}$-ray spectroscopy analysis showing the coexistence of $\mathrm{Fe}$ (Ka peak) and Au (La peak) in the nanoalloy. E-H) XANES spectra at the Fe- $\mathrm{K}$ edge (blue line in (E)) and at the Au- $\mathrm{L}_{\| 1}$ edge (blue line in $(\mathrm{F})$ ) of the $\mathrm{Au}_{0.89} \mathrm{Fe}_{0.11}$ nanoalloys, and the corresponding derivatives (blue lines in $(\mathrm{G})$ and $(\mathrm{H})$ for Fe and Au edges, respectively). The spectra of pure metal Fe (black lines) and Au (red lines) are also shown for comparison. The inset in panel $(E)$ shows an enlarged view of the low-energy region of the Fe-K edge.

structure) at the Au- $\mathrm{L}_{\mathrm{III}}$ edge, showing a slight decrease in the $\mathrm{Au}$-nearest neighbor distance with respect to the $\mathrm{Au}-\mathrm{Au}$ distance in pure bulk gold (Figure S1.2).

Information on the local electronic structure of Fe can be obtained by looking at the inset in Figure 1e, where the low energy part of the Fe K-edge spectrum is shown on an enlarged energy scale. This region is dominated by electronic transition to empty energy levels. In particular, the weak structures slightly above $7110 \mathrm{eV}$ are due to transitions from the $\mathrm{Fe} 1 \mathrm{~s}$ level to hybridized s,d bands, while the large shoulder in the absorption between 7111 and $7116 \mathrm{eV}$ is due to transitions to p-like states above the Fermi level. ${ }^{[39]}$ Comparison between the spectrum of the $\mathrm{Au}-\mathrm{Fe}$ nanoalloy and that of metallic Fe shows a marked decrease in the spectral weight for the transition to the p-like states for the alloy, while the transition to the s,d bands seems to be unaltered. Thus, a strong hybridization with Au states is deduced, lowering the density of the p-like states as projected on $\mathrm{Fe}$, reasonably due to large delocalisation effects.

Another key aspect of laser-generated nanoparticles is that they are not protected by any organic or undesired capping agent, which explains their excellent performances in recently reported catalytic applications. ${ }^{[19-22]} \mathrm{X}$-ray photoelectron analysis (Figure S1.3) shows that the surface of $\mathrm{Au}-$ Fe nanoalloys obtained from LASiS is composed only of metal $\mathrm{Au}$ and oxidized $\mathrm{Fe}$, constituting a passivation layer that confers to the nanoalloy excellent stability in air and water environment. ${ }^{[18]}$ The surface of the $\mathrm{Au}-\mathrm{Fe}$ nanoalloys is accessible for reactants and their catalytic activity can be investigated without any limitations or spurious effects owing to the protecting species. Taking advantage of the abovementioned peculiarities, in this work we investigated the electrochemical reactivity in alkaline aqueous environment $(1 \mathrm{M} \mathrm{KOH})$ of $\mathrm{Au}-\mathrm{Fe}$ alloy nanoparticles (NPs) with atomic composition $\mathrm{Au} 89$ at \% and $\mathrm{Fe} 11$ at \%, and compared their performances with other $\mathrm{Au}-\mathrm{Fe}$ alloy NPs containing a lower amount of iron ( 8 at \% and 3 at \%), as well as with pure gold NPs, iron oxide $\left(\mathrm{FeO}_{x}\right)$ NPs and a physical mixture of Au and $\mathrm{FeO}_{x}$ NPs, all of them obtained by the same LASiS protocol.

The electrocatalytic activity of the NPs was investigated by means of cyclic voltammetry $(\mathrm{CV})$ and electrochemical impedance spectroscopy (EIS). The experimental details on NP fabrication and the electrochemical experiments are reported in the Supporting Information (Chapters S1 and S2).

The CV of all samples supported on indium tin oxide (ITO) coated glass electrodes and tested in $1 \mathrm{~m} \mathrm{KOH}$ are shown in Figure $2 \mathrm{a}$. The $\mathrm{Au}_{0.89} \mathrm{Fe}_{0.11}$ NPs exhibit a remarkable improvement of OER activity in comparison to the $\mathrm{Au}$ and $\mathrm{FeO}_{x}$ NPs. The decrease of the iron content from 11 to 8 at $\%$ does not produce any relevant changes in the OER activity, while the behavior of $\mathrm{Au}_{0.97} \mathrm{Fe}_{0.03}$ is more similar to that of pure $\mathrm{Au}$ NPs. Figure $2 \mathrm{~b}$ shows a comparison of the relative values of overpotential $(\eta)$ required to achieve a current density of $10 \mathrm{~mA} \mathrm{~cm}^{-2}$, which is considered a relevant figure of merit in view of practical applications based on solar-tofuel conversion. ${ }^{[40]}$ In particular, the results reveal that $\mathrm{AuFe}$ NPs with the highest iron content ( 8 and 11 at \%) reach $10 \mathrm{mAcm}^{-2}$ at $\eta \approx 0.8 \mathrm{~V}$. Although still far from $\mathrm{IrO}_{2}$ and $\mathrm{RuO}_{2}\left(\eta_{\mathrm{J}=10 \mathrm{mAcm}^{-2}}=0.43\right),{ }^{[41]}$ which are the most efficient electrocatalysts for OER, this value is about $0.4 \mathrm{~V}$ lower than that obtained with $\mathrm{FeO}_{x}$ NPs $(1.17 \mathrm{~V})$ and even $1 \mathrm{~V}$ lower than that of pure Au NPs $(1.8 \mathrm{~V})$. This demonstrates that AuFe NP alloys incorporating an iron content well above the thermodynamic limit (8-11\%) offer a new route for enhancing the OER efficiency.

Figure $2 \mathrm{c}$ shows that the onset of OER is also significantly shifted to lower potential values for both $\mathrm{Au}_{0.89} \mathrm{Fe}_{0.11}$ and $\mathrm{Au}_{0.92} \mathrm{Fe}_{0.08}\left(\Delta E_{\text {onset }} \approx 0.1 \mathrm{~V}\right.$ in comparison to the other counterparts). $1.82 \mathrm{~V}$ vs. RHE has been chosen as reference potential for the following evaluations of current density, EIS measurements and accelerated stability tests, because at this potential value all the considered systems are under OER 

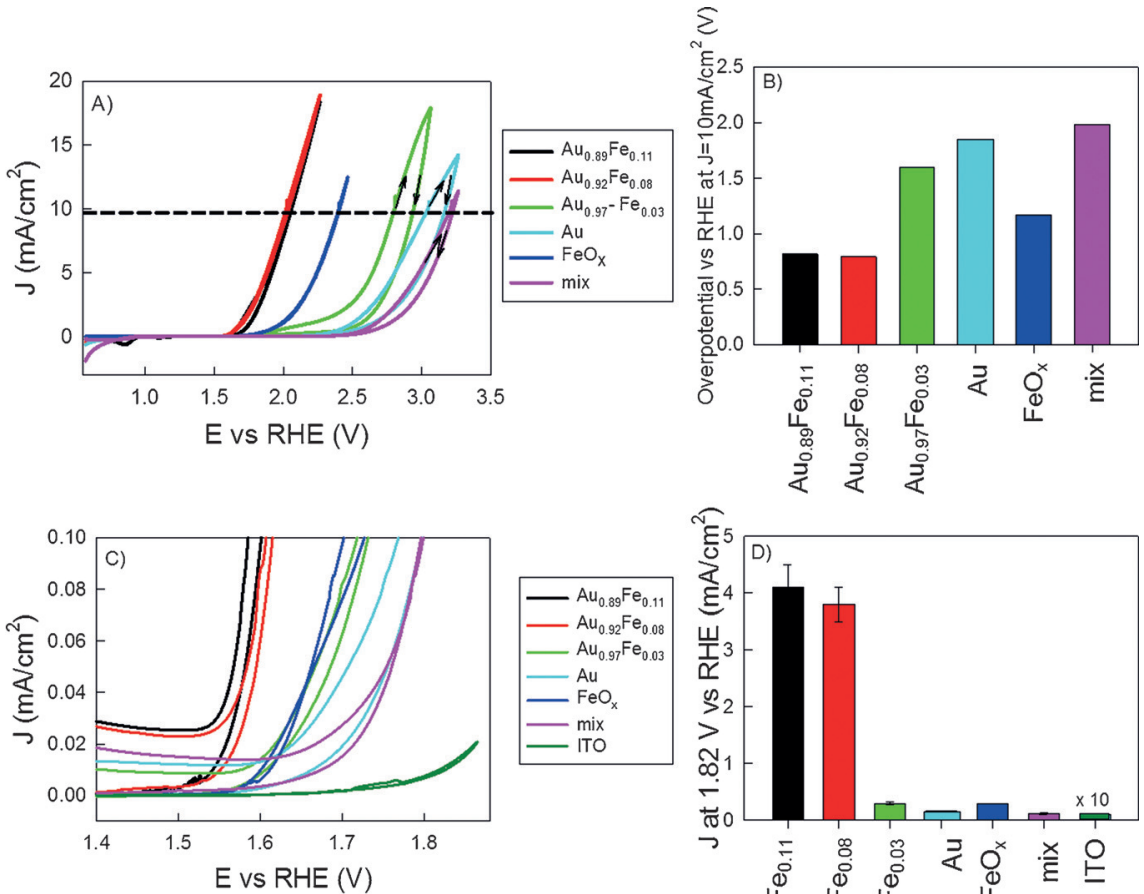

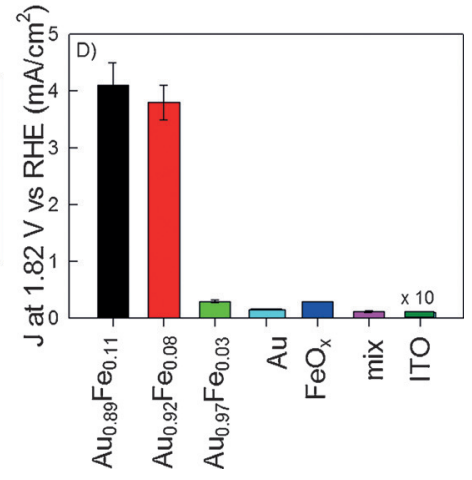

Figure 2. Comparison of cyclic voltammetry performed with different $\mathrm{Au}-\mathrm{Fe}$ nanoalloys, $\mathrm{Au}, \mathrm{FeO}_{x}$ and mixed $\mathrm{Au} / \mathrm{FeO}_{x} \mathrm{NPs}$ supported on ITO coated glass in $1 \mathrm{M} \mathrm{KOH}$. A) Cyclic voltammetry (CV) extended from 0 to $3.3 \mathrm{~V}$ vs. RHE, in order to obtain a current density $\mathrm{J}=10 \mathrm{mAcm}^{-2}$ for all the samples under analysis. B) Comparison of the overpotential required to achieve a current density $J=10 \mathrm{~mA} \mathrm{~cm}^{-2}$ extrapolated from the CV experiments reported in panel (A) for the different NPs. C) Zoom of the CV data in the potential range 1.4-1.87 V vs. RHE. The CV of naked ITO electrode is reported as a reference. D) Current density at $1.82 \mathrm{~V}$ vs. RHE. Data extrapolated from $\mathrm{CV}$ acquired from three independently prepared electrodes.

conditions. Figure $2 \mathrm{~d}$ shows that at $1.82 \mathrm{~V}$ the current density value of the alloy $\mathrm{Au}_{0.89} \mathrm{Fe}_{0.11}$ is ca. $4 \mathrm{mAcm}^{-2}$, about 23, 13 and 30 times higher than that of $\mathrm{Au}, \mathrm{FeO}_{x}$ and the physical mixture of $\mathrm{Au}$ and $\mathrm{FeO}_{\mathrm{x}} \mathrm{NPs}$, respectively. The turnover frequency (TOF), calculated according to Jaramillo and co-workers, ${ }^{[29]}$ was $0.09 \mathrm{~s}^{-1}$ at $1.82 \mathrm{~V}$. The current density reaches its maximum $\left(18.8 \mathrm{~mA} \mathrm{~cm}^{-2}\right)$ at $E=2.3 \mathrm{~V}$ vs. RHE, with a Faradaic yield $>95 \%$ (see Chapter S2). ${ }^{[25]}$

A further confirmation of the improved oxygen evolution activity of $\mathrm{Au}-\mathrm{Fe}$ nanoalloys resulted from electrochemical impedance spectroscopy analysis. Figure 3 shows Nyquist plots of $\mathrm{Au}_{0.89} \mathrm{Fe}_{0.11}, \mathrm{Au}, \mathrm{FeO}_{x}$ and the mixture of $\mathrm{Au} / \mathrm{FeO}_{x} \mathrm{NPs}$, upon acquisition between $1 \mathrm{~Hz}$ and $1 \mathrm{KHz}$ at $E=1.82 \mathrm{~V}$ (Figure $3 \mathrm{a}$ ) and at the equilibrium potential (Figure $3 b$ ).

EIS measurements were fitted according to the model reported in Figure 3, where Rct and Cdl correspond to the charge transfer resistance of the faradaic reaction and double layer capacitance at the interface electrode/electrolyte, respectively. Under OER conditions (Figure $3 \mathrm{a}$ ), the Rct value of $43 \Omega$ for $\mathrm{Au}-\mathrm{Fe}$ nanoalloys was remarkably lower than that of $\mathrm{Au}$ $(268 \Omega), \mathrm{FeO}_{x}(890 \Omega)$ and the physical mixture of $\mathrm{Au}$ and $\mathrm{FeO}_{x}(1070 \Omega) \mathrm{NPs}$, which indicates that the OER kinetics is faster for $\mathrm{Au}-\mathrm{Fe}$ nanoalloys, in agreement with data obtained from CV. EIS data acquired in OER conditions also show that the electrode/ electrolyte interface contribution dominates over diffusion.

EIS data acquired at equilibrium potential (Figure $3 \mathrm{~b}$ ) were used to calculate the electrochemical surface area (ECSA), according to the protocol proposed by Jaramillo
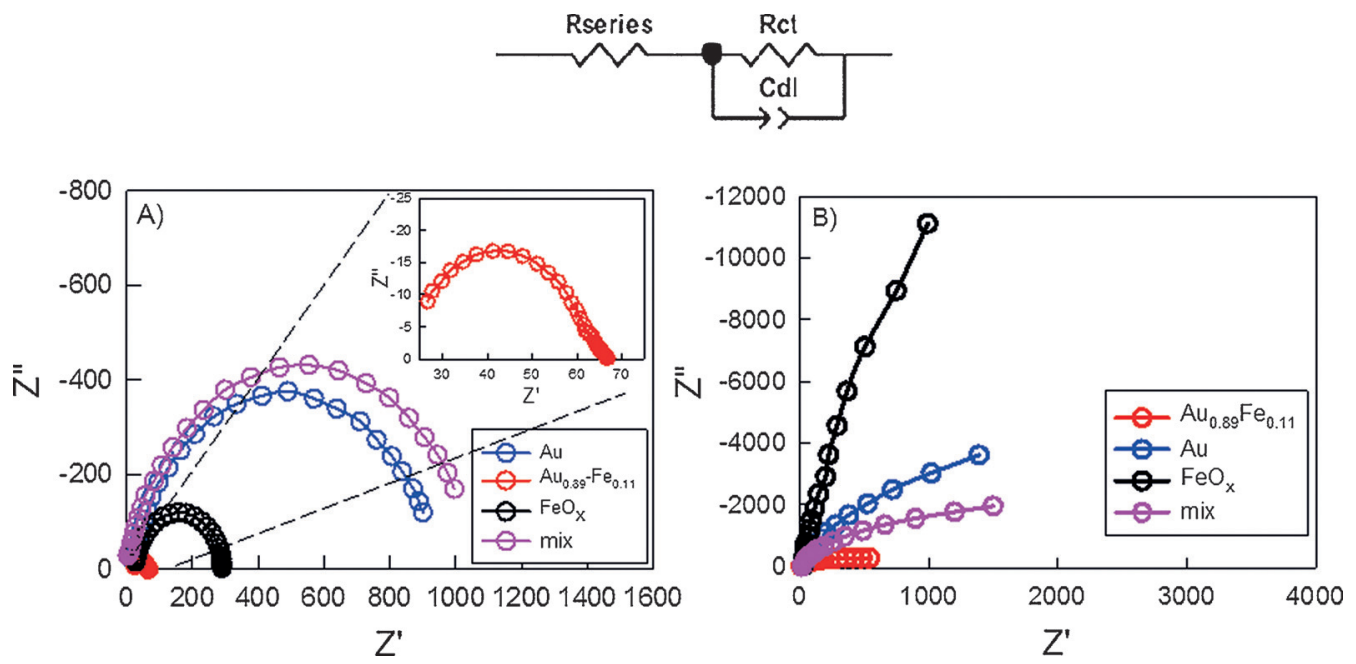

Figure 3. Nyquist plot obtained from EIS measurements performed A) under OER conditions (1.82 V vs. RHE) and B) at equilibrium potential. The model circuit utilized for fitting EIS data is shown on the top of the panel. The real and imaginary part of the impedance $Z$ are indicated as $Z$ ' and $Z^{\prime \prime}$, respectively. 
et al. ${ }^{[40]}$ For the $\mathrm{Au}_{0.89} \mathrm{Fe}_{0.11}$ nanoalloy an ECSA of $2.14 \mathrm{~cm}^{-2}$ has been obtained, which is much higher in comparison to that of bare $\mathrm{Au}\left(\mathrm{ECSA}=0.76 \mathrm{~cm}^{-2}\right), \mathrm{FeO}_{x}\left(\mathrm{ECSA}=0.31 \mathrm{~cm}^{-2}\right)$ and the physical mixture of the metals $\left(\mathrm{ECSA}=0.94 \mathrm{~cm}^{-2}\right)$. The related specific current densities at $E=1.82 \mathrm{~V}$ (see Chapter S3 for calculation details) show a similar trend.

The $\mathrm{Au}_{0.89} \mathrm{Fe}_{0.11}$ NPs were also subjected to a stress test of $1000 \mathrm{CV}$ cycles (scan rate: $300 \mathrm{mV} \mathrm{sec}^{-1}$ ) to investigate their stability (see Chapter S4). Under these conditions the maximum current progressively decreases to the $37 \%$ of its initial value. These data suggest that further efforts are required prior to the integration of this type of nanoalloys in realworking devices, as shown also from the tests performed with the configuration of a whole electrochemical cell, using a Nafion thin film as a membrane (see Chapter S5).

Overall, the experimental data indicate that the $\mathrm{Au}-\mathrm{Fe}$ nanoalloys result in an improved OER activity. OER mechanism is usually described to proceed from the initial adsorption of hydroxy ions that are discharged at the anode surface to form neutral $\mathrm{OH}$ adsorbates. Thus, depending on the type of catalyst, different steps may occur, involving the formation of a range of surface adsorbed intermediates, which concur to oxygen evolution at higher potential values. ${ }^{[35-37]}$ These steps strongly depend on the chemical nature of the catalyst. For example, a recent paper demonstrated that the OER activity of Ni-Fe layered double hydroxides is directly mediated by carbonate ions, which are formed by dissolution of $\mathrm{CO}_{2}$ in alkaline conditions and replace other interlayer anions. ${ }^{[37]}$

The analysis of Tafel plots, acquired at $0.2 \mathrm{mVs}^{-1}$ (see Chapter S6), reveals two distinct linear regions, which are commonly observed in literature. However, no specific hints on the origin of the improved OER can be inferred from Tafel analysis. ${ }^{[23,24]}$

The mutual interaction between $\mathrm{Au}$ and $\mathrm{Fe}$ (and other materials such as $\mathrm{Co}, \mathrm{Ni}$ or even $\mathrm{Mn}$ ) in OER catalysts has been the object of recent studies and is intensively investigated. ${ }^{[30,31]}$ Boettcher and co-workers reported that the OER activity of $\mathrm{FeOOH}$ films is strongly enhanced when they are deposited on Au electrodes. ${ }^{[14]}$ That study demonstrated that $\mathrm{FeOOH}$ is activated upon oxidation of $\mathrm{Au}$ to $\mathrm{AuO}_{x}$, which leads to the formation of hydrated $\mathrm{Au}-\mathrm{Fe}$ mixed surface oxides. Similar results were reported by Bell and co-workers for Fe impurities deposited on oxidized Au electrodes. ${ }^{[31]}$ All these studies point out the importance of mixing $\mathrm{Au}$ and $\mathrm{Fe}$ on the atomic (i.e. subnanometric) scale, where their chemical and electronic interaction is stronger. In particular, in order to improve OER activity, the conductivity of iron must be strongly enhanced, which means that the lattice surrounding the active species should be conductive. Gold is an ideal partner to develop synergistic interactions with iron. In a recent paper, Klaus et al. ${ }^{[15]}$ demonstrated that $\mathrm{Fe}^{3+}$ species, which are in the form of $\mathrm{FeO}_{2}^{-}$anions in alkaline $\mathrm{pH}$, can accumulate on the surface of oxidized gold and promote oxygen evolution through the conversion into ferrate(VI) ions $\left(\mathrm{FeO}_{4}{ }^{2-}\right)$. They also observed that the progressive removal of $\mathrm{Fe}^{3+}$ during the subsequent $\mathrm{CV}$ cycles leads to a decrease of the overall OER activity. The high reversibility of the redox state of $\mathrm{Fe}$ in the Au lattice of $\mathrm{Au}-\mathrm{Fe}$ nanoalloys has been also demonstrated in the recent study of Martirez and Carter. ${ }^{[16]}$

On the other hand, Karthik et al. ${ }^{[32]}$ demonstrated that the Fe species present in Fenton's reagent can be exploited to produce hydroxy radicals that remove low coordinated, metastable gold atoms from the surface of $\mathrm{Au}$ electrodes. As a result, the modified surface of the Au electrodes becomes much more efficient in promoting OER. Thus, depending on the system under analysis, the role of iron species can be different.

In this context, the production of nanoalloys that incorporate high amounts of iron can represent a real breakthrough for the development of a new class of electrocatalysts with unexplored performances, as well as to improve the current understanding about the role of transition metals in OER. ${ }^{[33]}$

These results show that alloying noble metals with transition metals is an appealing general approach to improve electrocatalytic oxygen evolution. It has been proposed that the same effect could be obtained also in other alloys of noble metals and transition metals with partially occupied d orbitals (such as $\mathrm{Co}$ and $\mathrm{Ni}$ ) ${ }^{[34]}$ which still deserve to be investigated. $^{[26-29]}$

\section{Acknowledgements}

We thank Dr. Mehwish Hassan (University of Brescia) for preliminary experiments and Prof. Stefano Agnoli (University of Padova) for XPS analysis. V.A. acknowledges financial support from University of Padova (PRAT no. CPDA114097/ 11).

\section{Conflict of interest}

The authors declare no conflict of interest.

Keywords: alkaline water electrolyzer .

electrochemical impedance spectroscopy · laser ablation .

nanoalloys $\cdot$ oxygen evolution reaction

How to cite: Angew. Chem. Int. Ed. 2017, 56, 6589-6593

Angew. Chem. 2017, 129, 6689-6693

[1] M. G. Walter, E. L. Warren, J. R. McKone, S. W. Boetcher, Q. Mi, E. A. Santori, N. S. Lewis, Chem. Rev. 2010, 110, 6446-6473.

[2] R. Subbaraman, D. Tripkovic, K.-C. Chang, D. Strmcnik, A. P. Paulikas, P. Hirunsit, M. Chan, J. Greeley, V. Stamenkovic, N. M. Markovic, Nat. Mater. 2012, 11, 550-557.

[3] W. T. Hong, M. Risch, K. A. Stoerzinger, A. Grimaud, J. Suntivich, Y. Shao-Horn, Energy Environ. Sci. 2015, 8, 14041427.

[4] L. Wang, J. Geng, W. Wang, C. Yuan, L. Kuai, B. Geng, Nano Res. 2015, 8, 3815-3822.

[5] B. M. Hunter, J. D. Blakemore, M. Deimund, H. B. Gray, J. R. Winkler, A. M. Müller, J. Am. Chem. Soc. 2014, 136, $13118-$ 13121.

[6] Y. Wu, M. Chen, Y. Han, H. Luo, X. Su, M.-T. Zhang, X. Lin, J. Sun, L. Wang, L. Deng, W. Zhang, R. Cao, Angew. Chem. Int. Ed. 2015, 54, 4870-4875; Angew. Chem. 2015, 127, 4952-4957. 
[7] M. Chen, Y. Wu, Y. Han, X. Lin, J. Sun, W. Zhang, R. Cao, ACS Appl. Mater. Interfaces 2015, 7, 21852-21859.

[8] G. Młynarek, M. Paszkiewicz, A. Radniecka, J. Appl. Electrochem. 1984, 14, 145-149.

[9] Y. Qiu, L. Xin, W. Li, Langmuir 2014, 30, 7893 - 7901.

[10] M. W. Louie, A. T. Bell, J. Am. Chem. Soc. 2013, 135, 1232912337.

[11] L. Trotochaud, S. L. Young, J. K. Ranney, S. W. Boettcher, J. Am. Chem. Soc. 2014, 136, 6744-6753.

[12] D. Friebel, M. W. Louie, M. Bajdich, K. E. Sawald, Y. Cai, A. M. Wise, M.-J. Cheng, D. Sokaras, T.-C. Weng, R. Alonso-Mori, R. C. Davis, J. R. Bargar, J. K. Nørskov, A. Nilsson, A. T. Bell, $J$. Am. Chem. Soc. 2015, 137, $1305-1313$.

[13] M. S. Burke, M. G. Kast, L. Trotochaud, A. M. Smith, S. W. Boettcher, J. Am. Chem. Soc. 2015, 137, 3638-3648.

[14] S. Zou, M. S. Burke, M. G. Kast, J. Fan, N. Danilovic, S. W. Boettcher, Chem. Mater. 2015, 27, 8011-8020.

[15] S. Klaus, L. Trotochaud, M.-J. Cheng, M. Head-Gordon, A. T. Bell, ChemElectroChem 2016, 3, 66-73.

[16] J. M. P. Martirez, E. A. Carter, ACS Nano 2016, 10, 2940-2949.

[17] H. Okamoto, Desk Handbook: Phase Diagrams for Binary Alloys, ASM International, Ohio, 2000.

[18] V. Amendola, S. Scaramuzza, S. Agnoli, S. Polizzi, M. Meneghetti, Nanoscale 2014, 6, 1423-1433.

[19] J. Zhang, M. Chaker, D. Ma, J. Colloid Interface Sci. 2017, 489, $138-149$.

[20] J. Zhang, G. Chen, D. Guay, M. Chaker, D. Ma, Nanoscale 2014, $6,2125-2130$

[21] S. Gu, J. Kaiser, G. Marzun, A. Ott, Y. Lu, M. Ballauff, A. Zaccone, S. Barcikowski, P. Wagener, Catal. Lett. 2015, 145, $1105-1112$.

[22] J. D. Blakemore, H. B. Gray, J. R. Winkler, A. M. Müller, ACS Catal. 2013, 3, 2497-2500.

[23] M. E. G. Lyons, R. L. Doyle, M. P. Brandon, Phys. Chem. Chem. Phys. 2011, 13, 21530-21551.

[24] M. E. G. Lyons, R. L. Doyle, Int. J. Electrochem. Sci. 2012, 7, 9488-9501.
[25] E. Pizzolato, S. Scaramuzza, F. Carraro, A. Sartori, S. Agnoli, V. Amendola, M. Bonchio, A. Sartorel, J. Energy Chem. 2016, 25, $246-250$.

[26] B. S. Yeo, A. T. Bell, J. Am. Chem. Soc. 2011, 133, 5587-5593.

[27] Y.-H. Fang, Z.-P. Liu, ACS Catal. 2014, 4, 4364.

[28] T. Shinagawa, A. T. Garcia-Esparza, K. Takanabe, Sci. Rep. $\mathbf{2 0 1 5}, 5,13801$.

[29] Y. Gorlin, C.-J. Chung, J. D. Benck, D. Nordlund, L. Seitz, T.-C. Weng, D. Sokaras, B. M. Clemens, T. F. Jaramillo, J. Am. Chem. Soc. 2014, 136, 4920-4926.

[30] R. Frydendal, M. Busch, N. B. Halck, E. A. Paoli, P. Krtil, I. Chorkendorff, J. Rossmeisl, Chem CatChem 2015, 7, 149-154.

[31] B. S. Yeo, S. L. Klaus, P. N. Ross, R. A. Mathies, A. T. Bell, ChemPhysChem 2010, 11, 1854.

[32] P. E. Karthik, C. Jeyabharathi, K. L. Phani, Chem. Commun. 2014, 50, 2787.

[33] Z. Xu, Y. Liu, F. Ren, F. Yang, D. Ma, Coord. Chem. Rev. 2016, $320-321,153-180$

[34] A. Holewinski, J.-C. Idrobo, S. Linic, Nat. Chem. 2014, 6, 828 834.

[35] B. M. Hunter, H. B. Gray, A. M. Müller, Chem. Rev. 2016, 116, $14120-14136$

[36] D. A. Corrigan, J. Electrochem. Soc. 1987, 134, 377-384.

[37] B. M. Hunter, W. Hieringer, J. R. Winkler, H. B. Gray, A. M. Müller, Energy Environ. Sci. 2016, 9, 1734-1743.

[38] F.-J. Lai, H.-L. Chou, L. S. Sarma, D.-Y. Wang, Y.-C. Lin, J.-F. Lee, B.-J. Hwang, C.-C. Chen, Nanoscale 2010, 2, 573-581.

[39] S. Kawata, K. Maeda, J. Phys. C 1978, 11, 2391-2397.

[40] C. C. L. McCRory, S. Jung, J. C. Peters, T. F. Jaramillo, J. Am. Chem. Soc. 2013, 135, 16977-16987.

[41] S. Jung, C. C. L. McCrory, I. M. Ferrer, J. C. Peters, T. F. Jaramillo, J. Mater. Chem. A 2016, 4, 3068-3076.

Manuscript received: March 31, 2017

Version of record online: May 2, 2017 